\title{
Multi-Period Investment Portfolio Selection of Interval Programming Based on Quantum Optimization Algorithm
}

\author{
Yongqi WU ${ }^{1}$, Tao $\mathrm{HAI}^{2}$, Hui ZHANG ${ }^{2 *}$ \\ ${ }^{1}$ Institute of Postgraduate Study, University of Malaya, 50603 Kuala Lumpur, Malaysia \\ 147816404@qq.com \\ ${ }^{2}$ School of Computer Science, Baoji University of Arts and Sciences, Baoji, 721000, China \\ haitao@bjwlxy.edu.cn, zhanghuisci@tom.com (*Corresponding author)
}

\begin{abstract}
Considering the portfolio selection problem in emerging markets which constantly face a serious shortage of information, the single-stage method of interval analysis can estimate assets income, assets risk, and approximate ranges of assets liquidity. However, it is difficult to evaluate flexibly and accurately assets income, assets risk, and approximate ranges of assets liquidity for multi-stage investment portfolio. In this paper, a novel quantum optimization model was applied to interval analysis method for multi-stage investment portfolio. Firstly, the multi-period investment portfolio selection models are designed by using inequalities equations of interval coefficients. Secondly, to solve the multi-stage investment portfolio constraint, the quantum mechanics is used along with an adaptive strategy designed to determine the best particle's positions for a better global position. Finally, the proposed constrained linear programming is validated by a practical example. Also, various decision-making factors were analysed for multi-period investment portfolio strategies which are compared with existing fuzzy portfolio selection models.
\end{abstract}

Keywords: Multi-period investment portfolio, Interval programming, Quantum optimization algorithm.

\section{Introduction}

The multi-period investment portfolio is focused on solving the allocating investors' wealth at hand reasonably, achieving a stable and rapid growth in capital investment and controlling the investment risks. Traditionally, the rebalancing problems of the portfolio during the investment period are not taken into consideration. It is assumed that investors will purchase a certain portfolio in their initial investment stage and hold it to the end of the investment period (Achour \& Rezg, 2007). However, in real life situations the investor will constantly rebalance the capital (that is buys and sells assets) in order to consider the portfolio issues as a multi-stage or dynamic adjustment process. In order to make their model be closer to the stock market situation, many scholars have expanded the portfolio optimization model into a multi-stage investment scenario under the single case of random uncertainty, taking consumption, taxes, transaction costs, inflation and other factors into consideration (Rădulescu \& Rădulescu, 2015).

Detemple (2014) formulated the mean-variance portfolio selection model as a two-standard optimization problem with the continuous time. The goal is to maximize the expected return on the terminal and minimize the variance of the terminal wealth. Forsyth et.al. (2012), study a continuoustime version of the proposed Markowitz mean- variance portfolio selection model and a market consisting of one bank account and multiple stocks is analyzed. The Markov regime conversion financial market was studied as a generalization of the model for individual investors with random liabilities (Kolm et.al., 2014). Dang, et.al., (2016) investigate the continuous-time meanvariance portfolio selection problem such as all market coefficients are random, and the wealth process under any acceptable trading strategy is not allowed to be below zero at any time. In Markowitz's portfolio selection model, the variance is replaced by a semi-variance. To that, a period portfolio option is extended to multiple periods. Yang et. al., (2015) originally proposed and developed a multi-cycle semi-variance model. The hybrid genetic algorithm (GA) is used to solve the multi-period and semi-variance model by using the displacement strategy of particle swarm optimization (PSO) as the mutation operation. Solving the multi-stage combinatorial optimization problem is a very challenging task due to its nonlinearity and its high computational time consumption. A number of heuristics have been employed to solve this problem. Zhang, et.al., (2012) propoesd a new particle swarm optimization algorithm, called drift particle swarm optimization algorithm and applied it for solving multi-stage combinatorial optimization problems. 
In all the above-mentioned portfolio selection models, it is assumed that the investors are facing random uncertainty, ignoring the vague uncertainty in the financial market. These models assume that the historical data on the assets from the financial market are available. However, sometimes due to the rapidly changing financial market the historical data may not be available. Liu et. al., (2013), study the concept of interval numbers and uses it to extend the classical mean-variance portfolio selection model with consideration of transaction cost to meanvariance-skewness model using fuzzy set theory. In addition, the future financial market is defined by three different models, pessimistically and in the combined form to model the fuzzy meanvariance-skewness portfolio selection problem. Aouni et al.( 2014) investigate several uncertain portfolio selection issues, where asset returns represented by interval data are discussed. Since the parameter is an interval value, the returned gain is also an interval value. The concept of the mean-absolute deviation function accordance with a two-level mathematical programming model is applied to compute the lower and upper limits of the investment returns of the portfolio selection problem. Using the dual theorem and applying the variable transformation technique, the twolevel mathematical program is converted into a traditional single-level mathematical program and the interval returned by the portfolio, which would cause problems for the math program, is solved. In addition, the probabilistic methods are used to detect the uncertainty in the financial market, and to determine the probability distribution of risk assets. However, the probability distribution function is often very difficult to predict accurately. Even if it is accurately predicted, it cannot guarantee that the return on assets follows this distribution. Many studies proposed the artificial intelligent in portfolio selection such as using the fuzzy mathematics. However, the study on the multi-portfolio problem is still in the exploratory stage. The investment time period decisions is always a multi-stage cases in the real market, so investors need to constantly adjust portfolios to achieve their investment intentions.

Since the proposed models are all programming models containing interval coefficients, the usual approach is to convert them into clear type models for the solution. In order to solve the proposed multi-period investment portfolio selection models, the interval programming method is used.
In this paper, the research is conducted by adopting interval analysis method, and establishing four optimized multi-period investment portfolio selection models. In the first section, the multiperiod investment portfolio selection models are designed by using inequalities equations of interval coefficients. The concept of possibility for denoting the inequality relationship of the interval numbers, and convert the interval coefficient inequality constraints involved in modelling into clear type inequalities is used. Secondly, the quantum particle swarm optimization algorithm is modelled for solving the converted models. Finally, in the experimental results, the actual data is used to analyze each decision-making factors and compare the existing selection of portfolio strategy with the proposed model.

\section{Multi-Period Investment Portfolio Selection Optimization Model of Interval Coefficient}

In an emerging market, as the historical information available to investors for reference is rather limited, it is usually very hard for investors to accurately provide precise values for the securities return. Under such conditions, investors can rely only on their own expertise and experience as well as on their sense of the market in order to estimate its rough fluctuation range. Under the above-mentioned conditions, this paper will utilize interval analysis method. Suppose that the return, risk and liquidity securities are all interval numbers. At the same time, suppose there are $n$ kinds of securities in the securities market available for transaction, and investors holding initial capital enter the market to plan an investment decision-making activity with a period of $\mathrm{T}$. To facilitate expression, relevant symbols involved in this paper will be described first:

$\tilde{r}_{i, t}=\left[\underline{r}_{i, t}, \bar{r}_{i, t}\right]$ refers to the yield rate of the $\mathrm{i}$ securities at period $\mathrm{t}$, where $0 \leq \underline{r}_{i, t} \leq \bar{r}_{i, t}$.

$\tilde{\delta}_{i, k}^{t}=\left[\underline{\delta}_{i, k}^{t}, \bar{\delta}_{(i, k)}^{t}\right]$ refers to the covariance between securities $\mathrm{i}$ and securities $\mathrm{k}$ at period $\mathrm{t}$, where $0 \leq \underline{\delta}_{i, k}^{t} \leq \bar{\delta}_{(i, k)}^{t} ; C_{t, i}$ refers to the rate of transaction expense collected for the transaction of securities $i$ at period $t$;

$x_{t}=\left(x_{t, 1}, x_{t, 2}, \ldots, x_{t, n}\right)$ refers to the investment ratio vector of period t stock portfolio, where $x_{t, i} \geq 0$ is the condition that forbids short selling; $\widetilde{R}_{p, t}$ refers to the interval income of period t stock portfolio; $\widetilde{R}_{N, t}$ refers to net

http://www.sic.ici.ro 
interval return of period t stock portfolio after deducting transaction costs; $W_{t}$ refers to the wealth gained by investors at the end of period $\mathrm{t}, t=1,2, \ldots, T$. In real investment process, the return and risk of securities are the two main factors considered by the investors. According to the above assumptions, the securities return $r_{i, t}$ and risk $\widetilde{\delta}_{i, k}^{t}$ are both interval numbers. Besides, as the investment rate vector is not a real number, i.e. invest $x_{t, i} \geq 0(i=1,2, \ldots, n ; t=1,2, \ldots, T)$. According to interval number operation rule, the return and risk of period $t$ stock portfolio are also interval numbers that can be expressed as follows:

$$
\begin{aligned}
& \widetilde{R}_{p, t}=\sum_{i=1}^{n} x_{t, i} \tilde{r}_{i, t}=\left[\sum_{i=1}^{n} x_{t, i} \underline{r}_{i, t}, \sum_{i=1}^{n} x_{t, i} \bar{r}_{i, t}\right] \\
& \tilde{\delta}\left(x_{t}\right)=\sum_{k=1}^{n} \sum_{i=1}^{n} x_{t, i} x_{t, k} \tilde{\delta}_{i, k}^{t}= \\
& {\left[\sum_{k=1}^{n} \sum_{i=1}^{n} x_{t, i} x_{t, k} \underline{\delta}_{i, k}^{t}, \sum_{k=1}^{n} \sum_{i=1}^{n} x_{t, i} x_{t, k} \bar{\delta}_{(i, k)}^{t}\right]}
\end{aligned}
$$

For transaction cost function, the commonly seen $\mathrm{V}$ function can still be used for expression, i.e. the total transaction cost of period $t$ stock portfolio can be expressed as:

$$
C_{t}=\sum_{i=1}^{n} c_{(t, i)}\left|x_{(t, i)}-x_{(t-1, i)}\right|, t=1,2, \ldots, T
$$

The net income of period $t$ stock portfolio after deducting transaction cost can be expressed as:

$W_{t}=W_{(t-1)} \prod_{j=2}^{t}\left[\sum_{i=1}^{n} x_{t, i} \tilde{r}_{i, t}-\sum_{i=1}^{n} c_{(t, i)}\left|x_{(t, i)}-x_{(t-1, i)}\right|\right]$

In Eq.(4), the terminal wealth that investors can get after $T$ investment can be expressed as:

$W_{T}=W_{0} \prod_{j=1}^{T}\left[\sum_{i=1}^{n} x_{t, i} \tilde{r}_{i, t}-\sum_{i=1}^{n} c_{(t, i)}\left|x_{(t, i)}-x_{(t-1, i)}\right|\right]=$

$\left[W_{0} \prod_{j=1}^{T} \sum_{i=1}^{n} x_{t, i} \underline{r}_{i, t}-\sum_{i=1}^{n} c_{(t, i)}\left|x_{(t, i)}-x_{(t-1, i)}\right|\right.$

$\left.W_{0} \prod_{j=1}^{T} \sum_{i=1}^{n} x_{t, i} \bar{r}_{i, t}-\sum_{i=1}^{n} c_{(t, i)}\left|x_{(t, i)}-x_{(t-1, i)}\right|\right]$

To simplify description, the upper and lower limits of terminal wealth can be expressed as:

$$
\begin{aligned}
& \underline{W}_{T}(x)=W_{0} \prod_{j=1}^{T}\left[\sum_{i=1}^{n} x_{t, i} \underline{r}_{i, t}-\sum_{i=1}^{n} c_{(t, i)}\left|x_{(t, i)}-x_{(t-1, i)}\right|\right] \\
& \bar{W}_{T}(x)=W_{0} \prod_{j=1}^{T}\left[\sum_{i=1}^{n} x_{t, i} \bar{r}_{i, t}-\sum_{i=1}^{n} c_{(t, i)}\left|x_{(t, i)}-x_{(t-1, i)}\right|\right]
\end{aligned}
$$

In addition to securities return, risk and skewness, liquidity of securities is also a key factor affecting investment decision. The measure of the liquidity of securities is important, being one of the key indicators reflecting the quality of the market operation. The so-called liquidity of securities refers to the quantity or amount of securities which can be bought or sold under current price situation or under lower price volatility circumstances. In actual investment decision-making process, illiquid securities will cause delays in the transaction, which in turn will cause investors to lose profit opportunities. Therefore, investors tend to select securities with good liquidity to invest on. The securities turnover rate is used to measure the liquidity. The securities turnover is assumed to be an interval number, and the securities turnover rate of the $\mathrm{i}$-th type of securities of period $\mathrm{t}$ is marked as $\tilde{l}_{(i, k)}^{t}=\left[\underline{l}_{(t, i)}, \bar{l}_{(t, i)}\right]$, in which, $0 \leq \underline{l}_{(t, i)} \leq \bar{l}_{(t, i)}$. Thus, the turnover rate of period $t$ stock portfolio is

$$
\tilde{l}\left(x_{t}\right)=\sum_{i=1}^{n} x_{t, i} l_{i, t}=\left[\sum_{i=1}^{n} x_{t, i} l_{(t, i)}, \sum_{i=1}^{n} x_{t, i} \bar{l}_{(t, i)}\right]
$$

The degree of securities diversification is another factor which affects investment decisions. Decentralized investment can reduce nonsystematic risk of the securities portfolio. The entropy ratio is used to measure the degree of portfolio diversification. Thus, the diversification of period $t$ stock portfolio can be expressed as

$$
H\left(x_{t}\right)=-\sum_{i=1}^{n} x_{t, i} \bar{l}_{(t, i)} \ln x_{(t, i)}, t=1,2, \ldots ., T
$$

In real life, an investment decision-making activity is often affected by many factors. Different investors take different decision-making factors into consideration. In order to simulate investing activities under different decision-making situations, this paper considers the rate of return, risk, liquidity, diversification of the portfolio and other factors, and formulates the following four different multi-period portfolio selection models of investment. The present analysis is based on the interval analysis.

Model 1: Let's suppose investors consider portfolio returns and risk as decision-making factors. The investor requires the expected portfolio returns of each period must to be greater than a given level. Another requirement is that the portfolio risk of each period must be lower than a given level. Under the above constraints, the investor seeks to find an investment-portfolio 
strategy which can maximize the portfolio terminal wealth. Thus, the following interval return-venture (RV) selection model of multiperiod investment portfolio selection $\left(P_{1}\right)$ is established. This formulation should be also used below for the similar sentences in the description of Models 2, 3 and 4.

$$
P_{1}\left\{\begin{array}{l}
\max W_{t}=\left[\underline{W}_{T}(x), \bar{W}_{T}(x)\right] \\
\text { s.t. }\left[\sum_{i=1}^{n} x_{t, i} \underline{r}_{i, t}, \sum_{i=1}^{n} x_{t, i} \bar{r}_{i, t}\right] \geq R_{t} \\
{\left[\sum_{k=1}^{n} x_{t=1} x_{t, k} \underline{\delta}_{i, k}^{t}, \sum_{k=1}^{n} \sum_{i=1}^{n} x_{t, i} x_{t, k} \bar{\delta}_{(i, k)}^{t}\right] \leq \delta_{t}} \\
\sum_{i=1}^{n} x_{t, i}=1 \\
x_{t, i} \geq 0, i=1,2 \ldots, n ; t=1,2, \ldots, T
\end{array}\right.
$$

in which $\mathrm{R}_{t}$ represents the investor's minimum required level on period $t$ stock portfolio return; $\delta_{t}$ represents the investor's maximum risk tolerance value on period $t$ stock portfolio. The constraint $x_{t, i} \geq 0$ shows that no short sales are allowed in the entire investment process.

$$
P_{2}\left\{\begin{array}{l}
\max W_{t}=\left[\underline{W}_{T}(x), \bar{W}_{T}(x)\right] \\
\text { s.t. }\left[\sum_{i=1}^{n} x_{t, i} \underline{r}_{i, t}, \sum_{i=1}^{n} x_{t, i} \bar{r}_{i, t}\right] \geq R_{t} \\
{\left[\sum_{k=1}^{n} x_{t, i} x_{t, k} \underline{\delta}_{i, k}^{t}, \sum_{k=1}^{n} \sum_{i=1}^{n} x_{t, i} x_{t, k} \bar{\delta}_{(i, k)}^{t}\right] \leq \delta_{t}} \\
{\left[\sum_{i=1}^{n} x_{t, i} l_{(t, i)}, \sum_{i=1}^{n} x_{t, i} \bar{l}_{(t, i)}\right] \geq l_{t}} \\
\sum_{i=1}^{n} x_{t, i}=1 \\
x_{t, i} \geq 0, i=1,2 \ldots, n ; t=1,2, \ldots, T
\end{array}\right.
$$

Model 2: Based on Model 1, it is assumed that the investor also requires portfolio liquidity of each period to be greater than a given level. Thus, the following return-venture-liquidity (RVL) multi-period investment portfolio selection model $\left(P_{2}\right)$ is established, in which $l_{t}$ represents the investor's minimum required level on the liquidity of period $t$ stock portfolio.

Model 3: Based on Model 1, the impact of multi-period stock portfolio diversification on the investment strategy is considered. Thus, the interval return-venture-entropy (RVE) multi- period investment portfolio selection model $\left(P_{3}\right)$ is established as follows:

$$
P_{3}\left\{\begin{array}{l}
\max W_{t}=\left[\underline{W}_{T}(x), \bar{W}_{T}(x)\right] \\
\text { s.t. }\left[\sum_{i=1}^{n} x_{t, i} \underline{r}_{i, t}, \sum_{i=1}^{n} x_{t, i} \bar{r}_{i, t}\right] \geq R_{t} \\
{\left[\sum_{i=1}^{n} x_{t, i} x_{t, k} \underline{\delta}_{i, k}^{t}, \sum_{k=1}^{n} \sum_{i=1}^{n} x_{t, i} x_{t, k} \bar{\delta}_{(i, k)}^{t}\right] \leq \delta_{t}} \\
-\sum_{i=1}^{n} x_{t, i} \bar{l}_{(t, i)} \ln x_{(t, i)} \geq e_{t} \\
\sum_{i=1}^{n} x_{t, i}=1 \\
x_{t, i} \geq 0, i=1,2 \ldots, n ; t=1,2, \ldots, T
\end{array}\right.
$$

in which $e_{t}$ represents the investor's minimum required level of the diversification of period $t$ stock portfolio.

Model 4: It assumed the investor considers all the factors involved in the three above-mentioned models. Thus, the interval return-venture-liquidityentropy (RVLE) multi-period investment portfolio selection model $\left(P_{4}\right)$ is established as follows:

$$
P_{4}\left\{\begin{array}{l}
\max W_{t}=\left[\underline{W}_{T}(x), \bar{W}_{T}(x)\right] \\
\text { s.t. }\left[\sum_{i=1}^{n} x_{t, i} \underline{r}_{i, t}, \sum_{i=1}^{n} x_{t, i} \bar{r}_{i, t}\right] \geq R_{t} \\
\left.\left[\sum_{k=1}^{n} \sum_{i=1}^{n} x_{t, i} x_{t, k} \underline{\delta}_{i, k}^{t}, \sum_{k=1}^{n} \sum_{i=1}^{n} x_{t, i} x_{t, k} \bar{\delta}_{(i, k)}^{t}\right] \leq \delta_{t} x_{t, i} l_{(t, i)}, \sum_{i=1}^{n} x_{t, i} \bar{l}_{(t, i)}\right] \geq l_{t} \\
-\sum_{i=1}^{n} x_{t, i} \bar{l}_{(t, i)} l n x_{(t, i)} \geq e_{t} \\
\sum_{i=1}^{n} x_{t, i}=1 \\
x_{t, i} \geq 0, i=1,2 \ldots, n ; t=1,2, \ldots, T
\end{array}\right.
$$

Note that the above four investment-portfolio models are all interval programming method problems. The interval number in the objective function represents the investor's gain of uncertain terminal wealth in his investment on the portfolio as follows:

In $\left[\sum_{i=1}^{n} x_{t, i} \underline{r}_{i, t}, \sum_{i=1}^{n} x_{t, i} \bar{r}_{i, t}\right] \geq R_{t}$, the left interval value represents the uncertain return of period $t$ portfolio; the $R_{t}$ on the right side represents the 
investor's minimum required level on the period t stock portfolio return;

$\operatorname{In}\left[\sum_{k=1}^{n} \sum_{i=1}^{n} x_{t, i} x_{t, k} \underline{\delta}_{i, k}^{t}, \sum_{k=1}^{n} \sum_{i=1}^{n} x_{t, i} x_{t, k} \bar{\delta}_{(i, k)}^{t}\right] \leq \delta_{t}$, the left interval value represents the uncertain risk of period t stock portfolio, and the $\delta_{t}$ on the right side represents the investor's maximum tolerance value on the risk of period $t$ stock portfolio;

$\operatorname{In}\left[\sum_{i=1}^{n} x_{t, i} l_{(t, i)}, \sum_{i=1}^{n} x_{t, i} \bar{l}_{(t, i)}\right] \geq l_{t}$, the left interval value represents the uncertain liquidity of period $\mathrm{t}$ stock portfolio, and the $1_{t}$ on the right side represents the investor's minimum required level on the liquidity of period $t$ stock portfolio.

To simplify the notation, in the following discussion, the feasible domains of $\left(P_{1}\right),\left(P_{2}\right)$, $\left(P_{3}\right),\left(P_{4}\right)$ as $x \in \Omega_{1}, x \in \Omega_{2}, x \in \Omega_{3}, x \in \Omega_{4}$ are separated.

In order to solve the objective function with constraint conditions by using multi-period investment portfolio selection models, the four models are transformed into the parameter planning problems, which are noted by $\left(P_{1}^{\prime}\right),\left(P_{2}^{\prime}\right),\left(P_{3}^{\prime}\right)$, and $\left(P_{4}^{\prime}\right)$.

Theorem 1: if $x^{*}$ is the optimal solution of $\left(P_{1}^{\prime}\right)$, then $\mathrm{x}^{*}$ is also the optimal solution of $P_{1}$.

Proof: Assume $\mathrm{x}^{*}$ is not the optimal solution of $P_{1}$, then the existence of $x \in \Omega_{1}$ makes $W_{T}(x) \geq W_{T}\left(x^{*}\right)$. Thus in $P_{1}^{\prime}$, the relationship $\lambda(x) \geq \lambda\left(x^{*}\right)$ is established. This is obviously in contradiction with the known conditions.

Similarly, problems $\left(P_{2}, P_{3}\right.$, and $\left.P_{4}\right)$ can be equivalently converted into the following parametric programming $P_{2}^{\prime}, P_{3}^{\prime}$ and $P_{4}^{\prime}$.

The above parametric programming $P_{1}^{\prime}, P_{2}^{\prime}, P_{3}^{\prime}$ and $P_{4}^{\prime}$ with interval coefficients, the interval coefficient inequality in the constraint condition indicates that this condition does not require strict fulfilment, which allows relaxation to a certain extent. Due to the partial order structure of interval number, usually there is no optimal solution in the classical sense.

The four models must be firstly converted into relevant clear-number type models. For all the interval coefficient inequalities in $P_{1}^{\prime}, P_{2}^{\prime}, P_{3}^{\prime}$ and $P_{4}^{\prime}$, a more frequently used approach is to convert inequalities containing interval number into clear-number type inequalities for the solution. This paper uses the possibility degree formula of interval number relationship to solve these interval inequality constraints.

For interval constraint inequalities

$W_{t}=\left[\underline{W}_{T}(x), \bar{W}_{T}(x)\right] \geq \lambda$

$\left[\sum_{i=1}^{n} x_{t, i} \underline{r}_{i, t}, \sum_{i=1}^{n} x_{t, i} \bar{r}_{i, t}\right] \geq R_{t}$

$\left[\sum_{i=1}^{n} x_{t, i} l_{(t, i)}, \sum_{i=1}^{n} x_{t, i} \bar{l}_{(t, i)}\right] \geq l_{t}$

Three above-mentioned interval coefficient inequalities can be separately converted into following clear-number type inequalities (Jiang et al., 2008). Based on an order relation of interval number, the uncertain function is transformed into two deterministic functions, in which the robustness of design is considered. Through a modified possibility degree, the uncertain inequality and equality constraints are changed to deterministic inequality constraints.

$P_{W_{T} \geq \lambda}=\frac{\bar{W}_{T}(x)-\lambda}{\bar{W}_{T}(x)-\underline{W}_{T}(x)} \geq \theta_{W_{T}} \rightarrow$

$\bar{W}_{T}(x)-\lambda \geq \theta_{W_{T}}\left(\bar{W}_{T}(x)-\underline{W}_{T}(x)\right)$

$P_{\widetilde{R}_{p, t} \geq R_{t}}=\frac{\sum_{i=1}^{n} x_{t, i} \bar{r}_{i, t}-R_{t}}{\sum_{i=1}^{n} x_{t, i} \bar{r}_{i, t}-\sum_{i=1}^{n} x_{t, i} \underline{r}_{i, t}} \geq \theta_{\widetilde{R}_{p, t}} \rightarrow$

$\sum_{i=1}^{n} x_{t, i} \bar{r}_{i, t}-R_{t} \geq \theta_{\widetilde{R}_{p, t}}\left(\sum_{i=1}^{n} x_{t, i} \bar{r}_{i, t}-\sum_{i=1}^{n} x_{t, i} \underline{r}_{i, t}\right)$

$P_{\tilde{l}\left(x_{t}\right) \geq l_{t}}=\frac{\left.\sum_{i=1}^{n} x_{t, i} \bar{l}_{(t, i)}\right]-l_{t}}{\sum_{i=1}^{n} x_{t, i} \bar{l}_{(t, i)}-\sum_{i=1}^{n} x_{t, i} l_{(t, i)}} \geq \theta_{\tilde{l}\left(x_{t}\right)} \rightarrow$

$\left.\sum_{i=1}^{n} x_{t, i} \bar{l}_{(t, i)}\right]-l_{t} \geq \theta_{i_{\left(x_{t}\right)}}\left(\sum_{i=1}^{n} x_{t, i} \bar{l}_{(t, i)}-\sum_{i=1}^{n} x_{t, i} l_{(t, i)}\right)$

where $\theta_{W_{T}}, \theta_{\widetilde{R}_{p, t}}, \theta_{\tilde{l}\left(x_{t}\right)} \in[0,1]$ represents the investor's acceptability on terminal wealth, period t portfolio return, and period t liquidity's degree of meeting the given standard.

For interval coefficient inequality

$\left[\sum_{k=1}^{n} \sum_{i=1}^{n} x_{t, i} x_{t, k} \underline{\delta}_{i, k}^{t}, \sum_{k=1}^{n} \sum_{i=1}^{n} x_{t, i} x_{t, k} \bar{\delta}_{(i, k)}^{t}\right] \leq \delta_{t}$ 
the following clear type inequality can be obtained according to (4):

$$
\begin{aligned}
& \delta_{t}-\sum_{k=1}^{n} \sum_{i=1}^{n} x_{t, i} x_{t, k} \underline{\delta}_{i, k}^{t} \geq \\
& \theta_{\tilde{\tilde{\delta}}\left(x_{t}\right)}\left[\sum_{k=1}^{n} \sum_{i=1}^{n} x_{t, i} x_{t, k} \bar{\delta}_{(i, k)}^{t}-\sum_{k=1}^{n} \sum_{i=1}^{n} x_{t, i} x_{t, k} \underline{\delta}_{i, k}^{t}\right]
\end{aligned}
$$

in which $\theta_{\tilde{\delta}\left(x_{1}\right)} \in[0,1]$ represents the investor's acceptability on period t portfolio's degree of meeting the given standard.

Based on the analyses above, if substituting Eqs. (12), (13), (14) into $P_{1}$, the following clear type optimized investment-portfolio model can be obtained

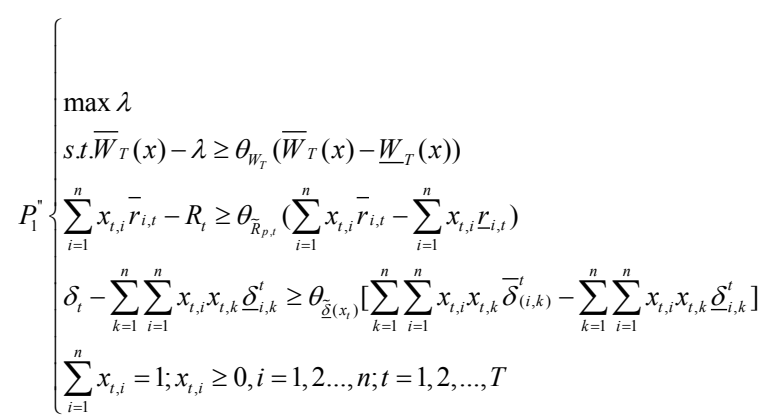

By substituting Eqs. (12), (13), (14) and (15) into $P_{2}$, the following clear type parametric programming can be obtained:

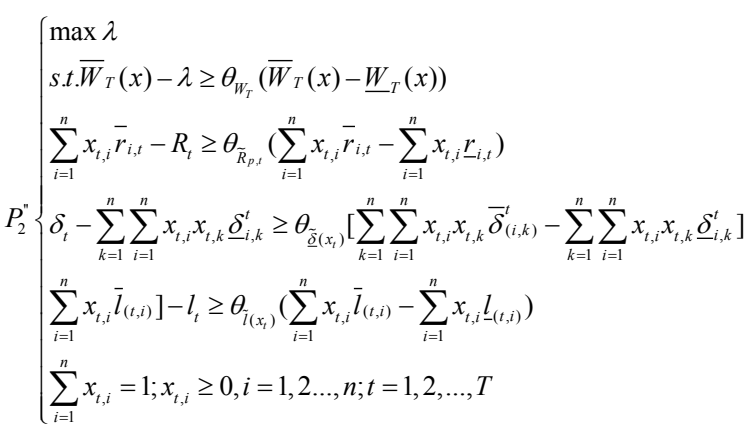

By substituting Eqs. (9), (10) and (12) into $P_{3}$, the following clear type parametric programming can be obtained:

$$
P_{3}\left\{\begin{array}{l}
\max \lambda \\
s . t \bar{W}_{T}(x)-\lambda \geq \theta_{W_{T}}\left(\bar{W}_{T}(x)-\underline{W}_{T}(x)\right) \\
\sum_{i=1}^{n} x_{t, i} \bar{r}_{i, t}-R_{t} \geq \theta_{\tilde{R}_{p, t}}\left(\sum_{i=1}^{n} x_{t, i} \bar{r}_{i, t}-\sum_{i=1}^{n} x_{t, i} \underline{r}_{i, t}\right) \\
\delta_{t}-\sum_{k=1}^{n} \sum_{i=1}^{n} x_{t, i} x_{t, k} \underline{\delta}_{i, k}^{t} \geq \theta_{\underline{\tilde{g}}(x, t)}\left[\sum_{k=1}^{n} \sum_{i=1}^{n} x_{t, i} x_{t, k} \bar{\delta}_{(i, k)}^{t}-\sum_{k=1}^{n} \sum_{i=1}^{n} x_{t, i} x_{t, k} \underline{\delta}_{i, k}^{t}\right] \\
-\sum_{i=1}^{n} x_{t, i} \bar{l}_{t(t, i)} l n x_{t(t, i)} \geq e_{t} \\
\sum_{i=1}^{n} x_{t, i}=1 ; x_{t, i} \geq 0, i=1,2 \ldots, n ; t=1,2, \ldots, T
\end{array}\right.
$$

By substituting Eqs. (10), (11), (12) and (13) into $P_{4}$ and it. the following clear type parametric programming can be obtained:

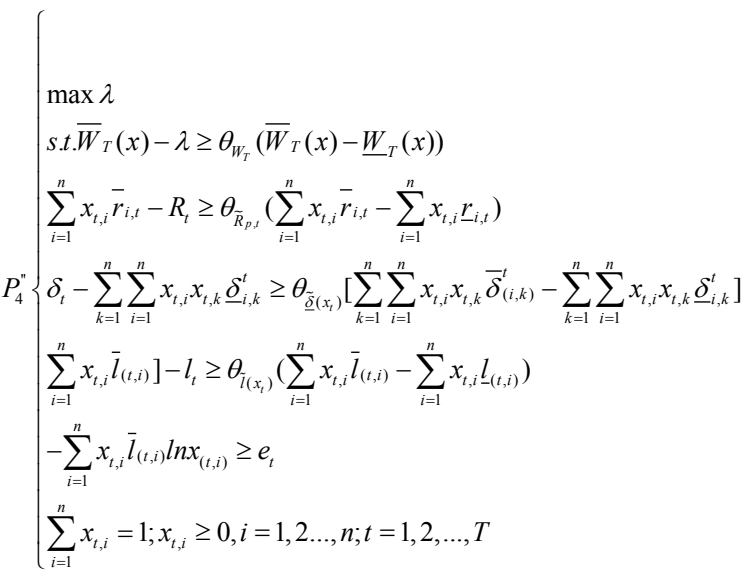

\section{Particle Swarm Algorithm Based on Quantum Behavior}

Particle swarm algorithm based on quantum behaviour refers to the best position that particles have experienced and, respectively, to the best position that all particles in the community have experienced. A new model of Particle swarm optimization (PSO) algorithm is used to improve the standard PSO from the perspective of quantum mechanics. This model is based on the DELTA potential well, believes particles have quantum behaviours, and based on this model, quantum particle swarm optimization algorithm (Alam et al., 2011) based on quantum behaviours was proposed. In quantum space, particles searching is available in the whole feasible solution space, thus Quantum-behaved particle swarm optimization (QPSO) algorithm is much better than the standard PSO algorithm in overall searching performance (Liu S. T., 2011). QPSO algorithm describes the state of the particles with waving function $\varphi(x, t)$ and obtains the probability density function of particles occurring at some point in the space by solving the Schrodinger equation. Then the equation of the particle's position is obtained through the Monte Carlo stochastic simulation as follows:

$$
X(t)=P \pm \frac{L}{2} \ln \left(\frac{1}{u}\right)
$$

where $\mathrm{u}$ is a random number distributed evenly on $[0,1]$; $\mathrm{L}$ is determined by equation $L(t)=2 \beta \mid$ mbest $-X(t) \mid$. The final evolution equation of the QPSO algorithm is: 


$$
\begin{aligned}
& \text { mbest }=\frac{1}{M} \sum_{i=1}^{M} p_{i}= \\
& \left(\frac{1}{M} \sum_{i=1}^{M} p_{i 1}, \frac{1}{M} \sum_{i=1}^{M} p_{i 2}, \ldots, \frac{1}{M} \sum_{i=1}^{M} p_{i D}\right) \\
& P_{i d}=\varphi \times p_{i d}+(1-\varphi) \times p_{g d} \\
& X_{i d}(t+1)= \\
& =p_{i d} \pm \beta \times \mid \text { mbestt }_{d}-X_{i d}(t) \mid \times \ln \left(\frac{1}{u}\right)
\end{aligned}
$$

where $\mathrm{M}$ is the number of particles in the community, D is the number of particles' dimensions, $u$ and $\varphi$ are uniformly distributed random numbers on $[0,1]$; , and mbest is the best position point of all the particles in the community. Same as standard PSO, $p_{i}$ and $p_{g}$ refer to the best position and, respectively, to the global position that all particles have in the community. $\beta$ is called contraction coefficient of expansion and is the only parameter of QPSO, and generally $\beta=0.5 \times($ Maxiter- $\mathrm{t}$ )/Maxiter +0.5 .

Due to its three characteristics, quantum particle swarm optimization can overcome the shortcomings of general particle swarm optimization in terms of convergence performance: (1) quantum systems have more states than linear systems. (2) The quantum system is an indeterminate system, and the particles have no clear trajectory. (3) In the PSO algorithm, the particles must be within a limited search range to ensure that the particle swarm clustering makes the algorithm converge at the best point or the local best point. In the PSO algorithm, a particle search range is limited, while in the QPSO algorithm particles can appear at a certain probability in any position of the whole feasible search space, even in a position far from the point.

The standard QPSO is not represented by the constraint mechanism, and therefore, it proves to be difficult to directly solve constrained programming problems. QPSO with constraint mechanism can be described by focusing on the following aspects:

- In order to control the evolution positions of the particle, a constraint factor $v$ was introduced in the position equation

$$
X_{i, d}^{G+1}=X_{i, d}^{G}+v V_{i, d}^{G+1}
$$

- In order to satisfy the constraint equation $\sum_{i=1}^{n} x_{t, i}=1$, in boundary constraints $\left(x_{i} \in\left[l_{i}, u_{i}\right], i=1,2, \ldots, n\right), \mathrm{N}$ particles were randomly generated. The particles were tested to satisfied equality constraints. If they are satisfied, then the particles are going to be used as initial particles. If they are not satisfied, then the particles are going to be transformed into $X_{G, i}^{,}=\frac{X_{G, i}}{\sum_{k=1}^{n} X_{G, k}}$.

- Supposed inequality constraints can be presented as $\omega_{n} \leq 0(n=1,2 \ldots, r)$, default values of generated particles can be calculated as $D(X)=\sum_{j=1}^{R} \max \left(\omega_{n}(X), 0\right)$.

- If $P_{i d}$ was not feasible and $X_{i, d}^{G+1}$ was feasible, $X_{i, d}^{G+1}$ replaced $P_{i d}$. If both $P_{i d}$ and $X_{i, d}^{G+1}$ were feasible with $f\left(X_{i, d}^{G+1}\right) \geq f\left(P_{i d}\right)$ , $X_{i, d}^{G+1}$ replaced $P_{i d}$. If neither $P_{i d}$ nor $X_{i, d}^{G+1}$ was feasible with $D\left(P_{i d}\right) \geq D\left(X_{i, d}^{G+1}\right), X_{i, d}^{G+1}$ replaced $P_{i d}$.

- Similarly, Gbest can also be processed.

\section{Experimental Results}

Since the stock market is the aggregation of buyers and sellers of stocks, which represent ownership claims on businesses, it's usually difficult to accurately estimate the return, risk and turnover rate of securities. To reduce the complexity, the investor selects four stocks from X Stock Exchange, and an analysis on the basis of the historical data of the weekly transaction's closing prices dating from January 2011 to January 2017 is conducted. For processing and analyzing these historical data, the investment period is set as a constant and the interval estimation method is used to compare with the proposed model, which was proposed by Bhattacharyya and Majumder (2011). In addition, to maximize terminal wealth expectations, it is better to consider the different period returns separately. Some investors may use their asset allocation on various periods. In this comparison, the period is set as 3 to reduce the complexity. Then, the return, turnover and covariance are obtained as shown in the followings in Tables 1, 2 and 3. 
Table 1. Interval Value Return of Each Period

\begin{tabular}{ccccc}
\hline t period & Stock 1 & Stock 2 & Stock 3 & Stock 4 \\
\hline 1 & {$[1.0070,1.0098]$} & {$[1.0060,1.0070]$} & {$[1.0105,1.0160]$} & {$[1.0252,1.0298]$} \\
2 & {$[1.0060,1.0080]$} & {$[1.0110,1.0130]$} & {$[1.0184,1.0250]$} & {$[1.0070,1.0097]$} \\
3 & {$[1.0165,1.0185]$} & {$[1.0260,1.0286]$} & {$[1.0225,1.0385]$} & {$[1.0120,1.0220]$} \\
\hline
\end{tabular}

Table 2. Interval Value Turnover of Each Period

\begin{tabular}{ccccc}
\hline t period & Stock 1 & Stock 2 & Stock 3 & Stock 4 \\
1 & {$[0.025,0.035]$} & {$[0.030,0.044]$} & {$[0.014,0.025]$} & {$[0.020,0.034]$} \\
2 & {$[0.018,0.036]$} & {$[0.012,0.066]$} & {$[0.030,0.075]$} & {$[0.025,0.045]$} \\
3 & {$[0.035,0.046]$} & {$[0.023,0.036]$} & {$[0.016,0.045]$} & {$[0.025,0.038]$} \\
\hline
\end{tabular}

Table 3. Interval Value Covariance of Each Period

\begin{tabular}{cccccc}
\hline t period & Stock & Stock 1 & Stock 2 & Stock 3 & Stock 4 \\
\hline 1 & Stock1 & {$[0.0278,0.0374]$} & {$[0.0213,0.0315]$} & {$[0.0228,0.0284]$} & {$[0.0141,0.0183]$} \\
& Stock2 & {$[0.0213,0.0315]$} & {$[0.0279,0.0346]$} & {$[0.0245,0.0356]$} & {$[0.0135,0.0218]$} \\
& Stock3 & {$[0.0228,0.0284]$} & {$[0.0245,0.0356]$} & {$[0.0266,0.0456]$} & {$[0.0178,0.0223]$} \\
& Stock4 & {$[0.0141,0.0183]$} & {$[0.01350 .0218]$} & {$[0.0178,0.0223]$} & {$[0.0215,0.0244]$} \\
2 & Stock1 & {$[0.0392,0.0592]$} & {$[0.0240,0.0352]$} & {$[0.0386,0.0483]$} & {$[0.0205,0.0363]$} \\
& Stock2 & {$[0.0240,0.0352]$} & {$[0.0226,0.0382]$} & {$[0.0202,0.0386]$} & {$[0.0252,0.0464]$} \\
& Stock3 & {$[0.0386,0.0483]$} & {$[0.0202,0.0386]$} & {$[0.0344,0.0518]$} & {$[0.0382,0.0585]$} \\
& Stock4 & {$[0.0205,0.0363]$} & {$[0.02520 .0464]$} & {$[0.0382,0.0585]$} & {$[0.0219,0.0257]$} \\
& Stock1 & {$[0.0208,0.0252]$} & {$[0.0277,0.0325]$} & {$[0.02430 .0301]$} & {$[0.02360 .0381]$} \\
& Stock2 & {$[0.0277,0.0325]$} & {$[0.0322,0.0447]$} & {$[0.0316,0.0473]$} & {$[0.0359,0.0542]$} \\
& Stock3 & {$[0.02430 .0301]$} & {$[0.0316,0.0473]$} & {$[0.0339,0.0469]$} & {$[0.0428,0.0556]$} \\
& Stock4 & {$[0.02360 .0381]$} & {$[0.0359,0.0542]$} & {$[0.0428,0.0556]$} & {$[0.0365,0.0511]$} \\
\hline
\end{tabular}

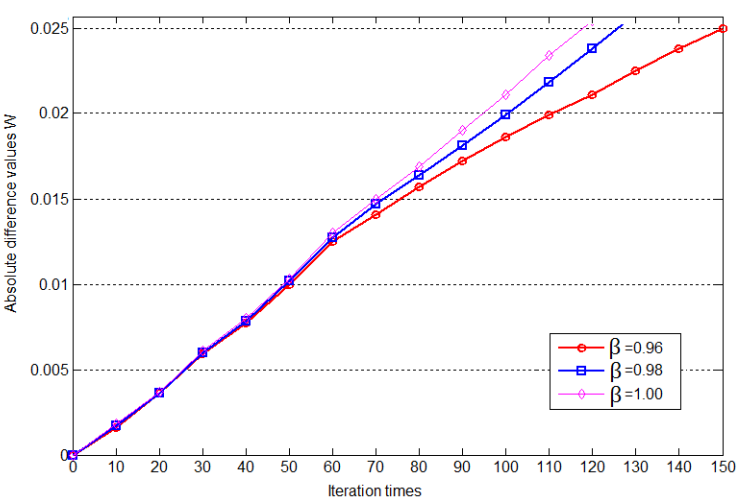

Figure 1. The different contraction coefficients comparison

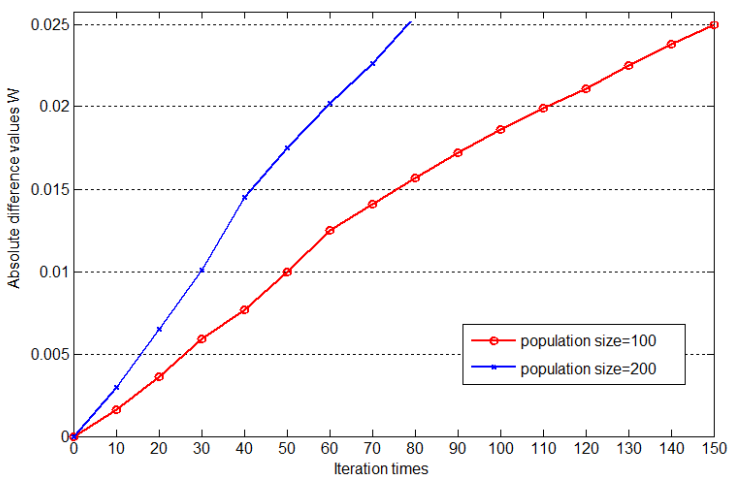

Figure 2. The different particles population comparison

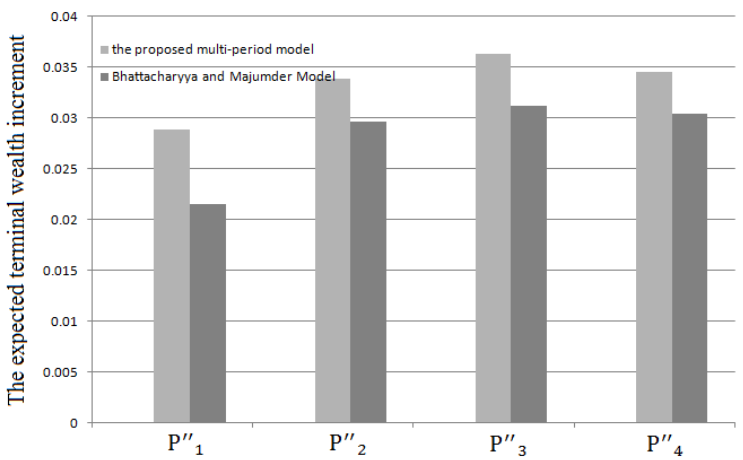

Figure 3. The expected terminal wealth increment comparison between the proposed multi-period model and the computation model of Bhattacharyya and Majumder

Convergence is an important characteristic of a stochastic optimization algorithm. In the Menger space, the fixed point theorem of the QPSO algorithm has proven that the algorithm converges to the global optimum in probability (Bhattacharyya \& Majumder, 2011). To analyse the convergence and evaluate the efficiency of the proposed algorithm, the period $\mathrm{P}_{4}{ }_{4}$ model has been set as a sample with 150 iteration times. The absolute 
Table 4. Optimal Investment Strategies in Various Decision Making Situations

\begin{tabular}{cccccc}
\hline Model & t period & Stock 1 & Stock 2 & Stock 3 & Stock 4 \\
\hline & 1 & 0.3233 & 0.0345 & 0.4427 & 0.1995 \\
$\mathrm{P} " 1$ & 2 & 0.0000 & 1.0000 & 0.0000 & 0.0000 \\
& 3 & 0.1236 & 0.0000 & 0.0000 & 0.8764 \\
& 1 & 0.2332 & 0.4333 & 0.0111 & 0.3224 \\
$\mathrm{P}$ "2 & 2 & 0.0000 & 0.0000 & 0.2841 & 0.7159 \\
& 3 & 0.4370 & 0.0000 & 0.0000 & 0.5630 \\
& 1 & 0.4319 & 0.0000 & 0.3515 & 0.2166 \\
$\mathrm{P}$ "3 & 2 & 0.0156 & 0.5649 & 0.4092 & 0.0103 \\
& 3 & 0.2756 & 0.0000 & 0.0618 & 0.6625 \\
& 1 & 0.2169 & 0.4566 & 0.0000 & 0.3266 \\
$\mathrm{P} 4$ & 2 & 0.0076 & 0.0472 & 0.2699 & 0.6752 \\
& 3 & 0.4457 & 0.0015 & 0.0252 & 0.5276 \\
\hline
\end{tabular}

Table 5. Multi-period Portfolio's Income /Risk /Liquidity Interval Corresponding to Each Investment Model

\begin{tabular}{|c|c|c|c|c|c|c|}
\hline Model & $t$ period & portfolio income & portfolio risk & portfolio liquidity & terminal wealth & terminal wealth expectations \\
\hline \multirow{3}{*}{$\mathrm{P}^{\prime \prime} 1$} & 1 & {$[1.0121,1.0164]$} & {$[0.0219,0.0301]$} & {$[0.0193,0.0307]$} & \multirow{3}{*}{$\begin{array}{l}{[1.0211,} \\
1.0336]\end{array}$} & \multirow{3}{*}{1.028842} \\
\hline & 2 & {$[1.0110,10.130]$} & {$[0.0226,0.0382]$} & {$[0.0262,0.0390]$} & & \\
\hline & 3 & {$[1.0126,1.0216]$} & {$[0.0335,0.0479]$} & {$[0.0262,0.0390]$} & & \\
\hline \multirow{3}{*}{$\mathrm{P}^{\prime \prime 2}$} & 1 & {$[1.0125,1.0151]$} & {$[0.0197,0.0268]$} & {$[0.0245,0.0385]$} & \multirow{3}{*}{$\begin{array}{c}{[1.0273,} \\
1.0405]\end{array}$} & \multirow{3}{*}{1.033908} \\
\hline & 2 & {$[1.0102,1.0140]$} & {$[0.0295,0.0411]$} & {$[0.0264,0.0535]$} & & \\
\hline & 3 & {$[1.0140,1.0205]$} & {$[0.0272,0.0398]$} & {$[0.0294,0.0415]$} & & \\
\hline \multirow{3}{*}{$\mathrm{P}^{\prime \prime} 3$} & 1 & {$[1.0122,1.0163]$} & {$[0.0218,0.0287]$} & {$[0.0201,0.0313]$} & \multirow{3}{*}{$\begin{array}{c}{[1.0208,} \\
1.0446]\end{array}$} & \multirow{3}{*}{1.036309} \\
\hline & 2 & {$[1.0139,1.0178]$} & {$[0.0239,0.0410]$} & {$[0.0196,0.0690]$} & & \\
\hline & 3 & {$[1.0139,1.0221]$} & {$[0.0307,0.0440]$} & {$[0.0272,0.0406]$} & & \\
\hline \multirow{3}{*}{$\mathrm{P}^{\prime \prime} 4$} & 1 & {$[1.0125,10151]$} & {$[0.0197,0.0268]$} & {$[0.0256,0.0388]$} & \multirow{3}{*}{$\begin{array}{c}{[1.0280} \\
1.0411]\end{array}$} & \multirow{3}{*}{1.034515} \\
\hline & 2 & {$[1.0103,1.0140]$} & {$[0.0290,0.0414]$} & {$[0.0257,0.0540]$} & & \\
\hline & 3 & {$[1.0143,1.0209]$} & {$[0.0272,0.0395]$} & {$[0.0292,0.417]$} & & \\
\hline
\end{tabular}

difference value $\mathrm{W}$ is used to clearly present the results with $\max \left|W_{T}-W_{0}\right|$. Figure 1 illustrates the results of different contraction coefficients comparison generated by $\beta=0.96,0.98$ and 1.00 . This means that for QPSO the convergence rate is faster than one of the PSO algorithm. With $\beta$ increasing, the rate of convergence increases for these three values. It is proved that the convergence of the function value is relatively difficult when the size is increased. For analysing the different particles population comparison, two groups of experiments were performed, one with population size $\mathrm{M}=100$, the other with $\mathrm{M}=200$. It should also be noted that for a given problem, when $\mathrm{M}=200$, the convergence speeds are larger than those when $\mathrm{M}=100$ in Figure 2.

However, it cannot be concluded that a larger population size results in a greater rate of convergence because the rate of convergence also depends on other parameters. Smaller population sizes may cause lower dimensional problems to converge faster, but when the dimensions are higher, the algorithm may encounter premature convergence. It can be inferred that when population size is increased to a certain number the convergence rate may exceed the convergence rate.

The algorithm parameters are as follows: inertia $\beta=0.98$; accelerating factor $\mathrm{c}_{1}=\mathrm{c}_{2}=1.49618$; maximum number of iterations $\mathrm{Gmax}=1000$, population size pop_size $=200$. Then, the Matlab optimization software for computing and the optimal investment strategy corresponding to each model after 1000 times of iterations will be used as seen in Table 4 .

Table 4 shows that by solving $P_{1}$, the corresponding investment strategies when the investor considers 
only the income and risk factors is obtained. In the decision making situation, the investor shall allocate the initiate wealth into these four stocks by these investment ratios: $32.33 \%, 3.45 \%, 44.27 \%$, $19.95 \%$; at the beginning of period 2 , he shall invest all his asset on stock 2; at the beginning of period 3, the investor shall invest his period-2end wealth on stock 1 and stock 4 by the ratios of $12.36 \%$ and $87.64 \%$. By solving $\left(P_{2}^{\prime \prime}, P_{3}^{\prime \prime}\right.$ and $\left.P_{4}^{\prime \prime}\right)$, the similar investment strategies can be obtained if the investor considers average value, risk and liquidity factors.

According to the investment strategies in various situations in Table 4, the multi-period portfolio's income, risk and the generally fluctuating range of liquidity corresponding to each investment model can be estimated.

The investment portfolio optimization problems in the emerging markets are related to the situation of insufficient information. It is supposed that investors can roughly estimate the security returns, risks and liquidity fluctuation ranges. The interval numbers are used to describe the uncertainty in the stock market. Four different multi-period portfolio selection models have been divided into 3 periods. The multi-period optimal investment strategies in various decisionmaking situations are shown in Table 4. The portfolio income interval, portfolio risk interval, liquidity interval and terminal wealth interval can be found within each investment period in each decision-making model, as shown in Table 5. In order to demonstrate the efficiency of the multiperiod investment portfolio selection model, the investor's expected terminal wealth indicators are applied to the comparison of the proposed multi-period model and the computation model of (Bhattacharyya \& Majumder, 2011). The expected terminal wealth increment comparison between two models has been presented in Figure 3. The expected terminal wealth increment i.e. the net income is expressed by the absolute difference between the expected terminal wealth and corporate initial wealth $\left(W_{0}\right)$. In addition, it indicates the investor's expected terminal wealth by the midpoint of portfolio terminal wealth.

Table 5 shows that the terminal wealth fluctuation range corresponding to problem ( $P_{1}$
) is [1.0211,1.0366], expected terminal wealth $W_{R V}=1.028842$, and net income is 0.028842 . In Figure 3, the expected terminal wealth increment is 0.022364 in the computation model (Bhattacharyya \& Majumder, 2011). The terminal wealth fluctuation range corresponding to problem $(P)$ is $[1.0273,1.0405]$, expected terminal wealth $\mathrm{W}_{\mathrm{RVL}}=1.033908$, and net income is 0.033908. In Figure 3, the expected terminal wealth increment is 0.029027 in the computation model (Bhattacharyya \& Majumder, 2011). The terminal wealth fluctuation range corresponding to problem $\left(P_{3}\right)$ is $[1.0280,1.0446]$, expected terminal wealth $W_{R V E}=1.036309$, and net income is 0.036309. In Figure 3, the expected terminal wealth increment is 0.031722 in the computation model (Bhattacharyya \& Majumder, 2011). The terminal wealth fluctuation range corresponding to problem $\left(P_{4}\right)$ is $[1.0280,1.0411]$, expected terminal wealth $\mathrm{W}_{\mathrm{RVE}}=1.034515$, and net income is 0.034515 . In Figure 3, the expected terminal wealth increment is 0.030041 in the computation model of Bhattacharyya and Majumder. For all the problems mentioned above $\left(P_{1}, P_{2}, P_{3}\right.$, and $\left.P_{4}\right)$, the net income is obviously lower than the one proposed in the multi-period model, as it can be seen in Figure 3. Thus, the proposed multi-period model is more efficient for obtaining the expected terminal wealth increment in comparison with the computation model (Bhattacharyya \& Majumder, 2011). Due to the introduction of possibility degree indicator, the investor's preferences on terminal wealth, multi-period portfolio income, risk, liquidity, and diversification level are more specific. By adjusting the possibility value of constraint validity of coefficient inequalities on each interval, the investor is enabled to accomplish his investment purpose.

Furthermore, by solving this four models, the inequality relationship between this four expected terminal wealth values is: $W_{R V}<W_{R V L}<W_{R V L E}<$ $W_{R V E}$. From the inequality relationship above, it's easy to find that the liquidity and diversification level of portfolio have a great impact on investment decision making factors; models which focus more on the liquidity and diversification of investment will be more effective than models which only focus on income and risk. 


\section{Conclusion}

Interval numbers are a special kind of fuzzy numbers, and intervals are powerful tools for dealing with uncertainty problems. Especially, when parameters of the probability density function cannot be obtained because of the lack of enough experimental data, the interval programming method is more practical than the analysis method of probability theory. The interval programming method is not only vague, i.e., the lack of sharp class boundaries, it also has a feature of uncertainty i.e., the lack of information can be addressed intuitively. This paper conducts the research about the multi-period investment portfolio selection problem in a booming stock market suffering of serious lack of information, fully considers decision making factors such as portfolio return, risk, liquidity and diversification level, and applies to interval number programming method to describe the uncertainty of the abovementioned decision making factors. The investor experience can approximately estimate the interval range of portfolio income, risk, liquidity and his market instinct. The interval programming method is used to argument according to these investment-portfolio optimization issues. Before modeling, some relevant interval number theories are involved. This paper conducts the adopting

\section{REFERENCES}

1. Achour, Z. \& Rezg, N. (2007). Time Floating General Mutual Exclusion Constraints, Studies in Informatics and Control, 16(1), 57-66.

2. Alam, S., Dobbie, G., Koh, Y. S., Riddle, P. \& Rehman, S. U. (2014). Research on particle swarm optimization based clustering: a systematic review of literature and techniques, Swarm and Evolutionary Computation, 17, 1-13.

3. Aouni, B., Colapinto, C. \& La Torre, D. (2014). Financial portfolio management through the goal programming model: Current state-of-the-art, European Journal of Operational Research, 234(2), 536-545.

4. Bhattacharyya, R., Kar, S. \& Majumder, D. D. (2011). Fuzzy mean-variance-skewness portfolio selection models by interval analysis, Computers \& Mathematics with Applications, 61(1), 126-137. interval analysis method, and establishes four optimized multi-period investment portfolio selection models by using the concept of possibility of denoting the inequality relationship of interval numbers. The constrained linear programming method converts the interval coefficient inequality constraints involved in modeling into clear type inequalities. Furthermore, because the particle swarm optimization algorithm is not efficient for the constrained linear programming and for a global convergence, the strategy of a double exponential distribution and an adaptive method to sample particle's positions is designed for multiperiod investment portfolio selection models with interval analysis. Finally, the proposed model is tested and compared with each decision-making factor's impact on the selection of portfolio strategy. The experimental results show that, at a certain acceptable level of possibility, taking into consideration the investment diversification and the impact of liquidity will lead to a greater terminal wealth than the one obtained considering only the income and risk factors. Compared with the existing portfolio strategy, if the investor is dissatisfied with the investment strategy obtained, multi-period investment portfolio selection can be used to get optimal personal investment strategy by adjusting acceptability level value.

5. Dang, D. M., Forsyth, P. A. \& Li, Y. (2016). Convergence of the embedded mean-variance optimal points with discrete sampling, Numerische Mathematik, 132(2), 271-302.

6. Detemple, J. (2014). Portfolio Selection: A Review, Journal of Optimization Theory and Applications, 161(1), 1-21.

7. Forsyth, P. A., Kennedy, J. S., Tse, S. T. \& Windcliff, H. (2012). Optimal trade execution: a mean quadratic variation approach, Journal of Economic Dynamics and Control, 36(12), 1971-1991.

8. Jiang, C., Han, X., Liu, G. R. \& Liu, G. P. (2008). A nonlinear interval number programming method for uncertain optimization problems, European Journal of Operational Research, 188(1), 1-13. 
9. Kolm, P. N., Tütüncü, R. \& Fabozzi, F. J. (2014). 60 Years of portfolio optimization: Practical challenges and current trends, European Journal of Operational Research, 234(2), 356-371.

10. Liu, S T. (2011). The mean-absolute deviation portfolio selection problem with interval-valued returns, Journal of Computational and Applied Mathematics, 235(14), 4149-4157.

11. Liu, Y. J., Zhang, W. G. \& Zhang, P. (2013). A multi-period portfolio selection optimization model by using interval analysis, Economic Modelling, 33, 113-119.
12. Rădulescu, M. \& Rădulescu, C. Z. (2015). A Portfolio Theory Approach to Software Vendor Selection, Studies in Informatics and Control, 24(4), 380. doi:10.24846/v24i4y201502

13. Yang, Z., Yin, G. \& Zhang, Q. (2015). Mean-variance type controls involving a hidden Markov chain: models and numerical approximation, IMA Journal of Mathematical Control and Information, 32(4), 867-888.

14. Zhang, W. G., Liu, Y. J. \& Xu, W. J. (2012). A possibilistic mean-semivariance-entropy model for multi-period portfolio selection with transaction costs, European Journal of Operational Research, 222(2), 341-349. 